\title{
Mycotoxins, in a Prospection in the Causes and Treatment of Metabolic Syndrome
}

\begin{abstract}
Metabolic syndrome (MS) and MS-related phenomena are widely distributed. For examples, cardiovascular disease and type 2 diabetes are two common diseases known to occur in MS. Till date, a number of risk factors as well as treatment strategies have been introduced in MS. Mtcotoxins are one of them. This paper features mycotoxins as a risk factor as well as therapeutic tools of MS.
\end{abstract}

Keywords: Mycotoxins; Metabolic syndrome; Prospection

Mini Review
Volume 8 Issue 6 - 2017
Muhammad Torequl Islam*
Federal University of Piaui, Brazil
*Corresponding author: Muhammad Torequl Islam, Post-
Doctorate Researcher, Federal University of Piaui, Teresina
(PI)-64.049-550, Brazil, Email: rbiotufpi.br@gmail.com
Received: September 11, 2017 | Published: September 21,
2017

Abbreviations: CVD: Cardiovascular Disease; MS: Metabolic Syndrome; HDL: High-Density Lipoprotein; NDDs: Chronic Neurodegenerative Diseases; LPS: Lipopolysaccharides; NTIS: Non-Thyroidal Illness Syndrome

\section{Introduction}

Abdominal (central) obesity, elevated blood pressure, elevated fasting plasma glucose, high serum triglycerides and low highdensity lipoprotein (HDL) levels are the key features of the metabolic syndrome (MS) that is associated with the risk of developing cardiovascular disease (CVD) and type 2 diabetes [1].

Various strategies have been proposed to prevent/management the development of MS. Among them, changing of lifestyle and food habit is most common. A number of drug therapies are also recommended to treat or control of MS. To date, a number of risk factors also identified for the development of MS. Mycotoxins, toxic secondary metabolites produced by fungus kingdom [2] are not only evident to cause MS but also helpful to treat MS. This article depicts a perspection on myotoxins in MS.

\section{Methodology}

A search was made in the PubMed database for published articles on the following keywords: mycotoxins in metabolic syndrome; mycotoxin-induced metabolic syndrome; mycotoxins in the treatment of metabolic syndromes. Articles from the past to till date, concerning the search topic was collected and used for this review. Articles falling outside the scope of this study were discarded by reading the titles, abstracts and contents.

\section{Mycotoxins Causing MS}

Aflatoxin, in chicks showed a strong malabsorption syndrome characterized by steatorrhea, hypocarotenoidemia, and decreased concentrations of bile salts and pancreatic lipase, trypsin, amylase, and RNase, while T-2 characterized by steatorrhea and decreased levels of pancreatic lipase, trypsin, amylase, and RNase [3]. In the same study, ochratoxicosis was evident to show severe hypocarotenoidemia. Though, T-2 toxicosis exhibited lipid malabsorption in the absence of hypocarotenoidemia, ochratoxicosis exhibited hypocarotenoidemia in the absence of lipid malabsorption, but aflatoxicosis exhibited both symptoms.
In low content of vitamin $\mathrm{E}(6 \mathrm{mg} / \mathrm{kg}$ as compared with 100 $\mathrm{mg} / \mathrm{kg}$ in control), T-2 mycotoxin in rats decreased in activity of lysosomal enzymes, aniline hydroxylase, carboxyl esterase and in content of cytochrome P-450 in liver tissue simultaneously with twofold activation of epoxide hydrolase and UDP-glucuronosyl transferase; decreased in non-sedimented activity of lysosomal enzymes; decreased in activity of alkaline phosphatase and of lysosomal enzymes in blood serum [4].

The role of large-conductance $\mathrm{Ca}^{2+}$-activated $\mathrm{K}^{+}$(BK (Ca)) channels in the regulation of coronary microvascular function is widely appreciated. In a study, male Ossabaw miniature swine consumed in 3-6 mo a normal diet (11\% kcal from fat) or an excess-calorie atherogenic diet that induces MetS (45\% kcal from fat, $2 \%$ cholesterol, $20 \% \mathrm{kcal}$ from fructose). MetS significantly impaired coronary vasodilation to the BK(Ca) opener NS-1619 in vivo $(30-100 \mu \mathrm{g})$ and reduced the contribution of these channels to adenosine-induced microvascular vasodilation in vitro (1 $100 \mu \mathrm{M})$. MetS reduced whole cell penitrem A (1 $\mu \mathrm{M})$-sensitive $\mathrm{K}^{+}$current and NS-1619-activated $(10 \mu \mathrm{M})$ current in isolated coronary vascular smooth muscle cells. MetS increased the concentration of free intracellular $\mathrm{Ca}^{2+}$ and augmented coronary vasoconstriction to the L-type $\mathrm{Ca}^{2+}$ channel agonist BAY K 8644 (10pM - 10nM). BK (Ca) channel alpha and beta (1) protein expression was increased in coronary arteries from MetS swine. Coronary vascular dysfunction in MetS is related to impaired BK (Ca) channel function and is accompanied by significant increases in L-type $\mathrm{Ca}^{2+}$ channel-mediated coronary vasoconstriction [5]. In another study of this research group, penitrem A $(10 \mu \mathrm{g} / \mathrm{kg}$, i.v) revealed that the exercise-induced increases in blood pressure were significantly elevated in MetS swine [6].

Chronic neurodegenerative diseases (NDDs) are associated with obesity and diabetes worldwide, mainly the Parkinson's and Alzheimer's disease. MS involves lipoprotein abnormalities and insulin resistance is the major cause of induction of NDDs. The effects of bacterial lipopolysaccharides (LPS) on dyslipidemia 
and NAFLD indicate that the clearance and metabolism of fungal mycotoxins are linked to hypercholesterolemia and amyloid beta oligomers. LPS and mycotoxins are associated with membrane lipid disturbances with effects on cholesterol interacting proteins, lipoprotein metabolism, and membrane apo E/amyloid beta interactions relevant to hypercholesterolemia with close connections to NDDs [7].

Long-term exposure to dampness microbiota induces multiorgan morbidity. One of the symptoms related to this disorder is non-thyroidal illness syndrome (NTIS). A retrospective study was carried out in nine patients with a history of mold exposure, experiencing chronic fatigue, cognitive disorder, and different kinds of hypothyroid symptoms. Mycotoxin exposure via the diet is underlined as DIO2 genetic polymorphism and dysfunction of DIO2 play an important role in the development of symptoms [8].

\section{Mycotoxins Against MS}

Drugs or products acting as chemo-modulators were assayed for their effect on the chemotactic activity. Cytochalasin B is evident by interfering with the cytoskeletal elements (microtubules and microfilaments) cyclic nucleotides involved in the metabolic activity during the cell motility [9]. In another study, AFB1 (0.1, 0.3 or $1 \mu \mathrm{g} / \mathrm{mL}$ ) in Li-Fraumeni patient, but not cells from normal individuals, can induce immortalization [10].

\section{Conclusion}

Mycotoxins are one of the important causes of MS. However, some MS along with the related phenomena could be taken into account that there is an option to select mycotoxins and their laboratory derivatives to be treated. Mycotoxins are important lead compounds in MS and MS-related complications.

\section{Conflict of Interest}

None declared.

\section{References}

1. Kaur J (2014) A comprehensive review on metabolic syndrome. Cardiol Res Practice 2014: 943162

2. Richard JL (2007) Some major mycotoxins and their mycotoxicosesan overview. Int J Food Microbiol 119(1-2): 3-10.

3. Osborne DJ, Huff WE, Hamilton PB, Burmeister HR (1982) Comparison of ochratoxin, aflatoxin, and T-2 toxin for their effects on selected parameters related to digestion and evidence for specific metabolism of carotenoids in chickens. Poult Sci 61(8): 1646-1652.

4. Kravchenko LV, Kranauskas AE, Dzhaparidze LM, Avren'eva LI, et al. (1986) Effect of different supplies of vitamin $E$ on biochemical changes in T-2 mycotoxicosis in rats. Vopr Med Khim 32(6): 99-103.

5. Borbouse L, Dick GM, Asano S, Bender SB, Dincer UD, et al. (2009) Impaired function of coronary $\mathrm{BK}(\mathrm{Ca})$ channels in metabolic syndrome. Am J Physiol Heart Circ Physiol 297(5): H1629-H1637.

6. Borbouse L, Dick GM, Payne GA, Payne BD, Svendsen MC, et al. (2010) Contribution of BK(Ca) channels to local metabolic coronary vasodilation: Effects of metabolic syndrome. Am J Physiol Heart Circ Physiol 298(3): H966-H973.

7. Martins IJ (2015) Overnutrition Determines LPS Regulation of Mycotoxin Induced Neurotoxicity in Neurodegenerative Diseases. Int J Mol Sci 16(12): 29554-29573.

8. Somppi TL (2017) Non-Thyroidal Illness Syndrome in Patients Exposed to Indoor Air Dampness Microbiota Treated Successfully with Triiodothyronine. Front Immunol 8: 919.

9. Pham Huu T (1979) Chemotaxis of human leucocytes: II. Effects of lectins, colchicine, cytochalasin B, cyclic nucleotides and immunostimulatory products. Biomedicine 30(2): 121-124.

10. Tsutsui T, Fujino T, Kodama S, Tainsky MA, Boyd J, et al. (1995) Aflatoxin B1-induced immortalization of cultured skin fibroblasts from a patient with Li-Fraumeni syndrome. Carcinogenesis 16(1): $25-34$ 